\title{
IGL-1 solution reduces endoplasmic reticulum stress and apoptosis in rat liver transplantation
}

\author{
IB Mosbah ${ }^{1}$, MA Zaouali ${ }^{1}$, C Martel ${ }^{2}$, M Bjaoui ${ }^{1,3}$, HB Abdennebi ${ }^{3}$, G Hotter ${ }^{1}$, C Brenner ${ }^{\star, 2,4}$ and J Roselló-Catafau ${ }^{1,4}$
}

Injury due to cold ischemia reperfusion (I/R) is a major cause of primary graft non-function following liver transplantation. We postulated that $\mathrm{I} / \mathrm{R}$-induced cellular damage during liver transplantation might affect the secretory pathway, particularly at the endoplasmic reticulum (ER). We examined the involvement of ER stress in organ preservation, and compared cold storage in University of Wisconsin (UW) solution and in Institute Georges Lopez-1 (IGL-1) solution. In one group of rats, livers were preserved in UW solution for $8 \mathrm{~h}$ at $4{ }^{\circ} \mathrm{C}$, and then orthotopic liver transplantation was performed according to Kamada's cuff technique. In another group, livers were preserved in IGL-1 solution. The effect of each preservation solution on the induction of ER stress, hepatic injury, mitochondrial damage and cell death was evaluated. As expected, we found increased ER stress after liver transplantation. IGL-1 solution significantly attenuated ER damage by reducing the activation of three pathways of unfolded protein response and their effector molecules caspase-12, C/EBP homologous protein-10, X-box-binding protein 1, tumor necrosis factor-associated factor 2 and eukaryotic translation initiation factor 2 . This attenuation of ER stress was associated with a reduction in hepatic injury and cell death. Our results show that IGL-1 solution may be a useful means to circumvent excessive ER stress reactions associated with liver transplantation, and may optimize graft quality.

Cell Death and Disease (2012) 3, e279; doi:10.1038/cddis.2012.12; published online 8 March 2012

Subject Category: Experimental Medicine

Cells, tissues and organs that are routinely transplanted are often exposed to environmental stress. Hypothermia is believed to protect cells by slowing their metabolism during hypoxic storage. But it also causes injury, which is aggravated by all classically used preservation solutions. ${ }^{1}$ Injury induced by hypothermia is a major cause of primary graft non-function following liver transplantation. ${ }^{2}$ However, the introduction of University of Wisconsin (UW) solution improved graft and patient survival. ${ }^{3,4}$ This liquid, considered as the gold standard of preservation solution, has proved effective in preventing organ damage during cold ischemia and has extended the storage time. ${ }^{3,4}$ However, UW solution does have certain properties that hinder organ preservation, such as its increased viscosity and the presence of hydroxyethyl starch (HES). ${ }^{5,6}$ Recently, we have described Institute Georges Lopez-1 (IGL-1) preservation solution as an effective alternative to UW solution. ${ }^{7,8} \mathrm{IGL}-1$ composition is characterized by the reversion of the ionic $\mathrm{K} / \mathrm{Na}$ concentrations and the replacement of HES by polyethylene glycol 35 (PEG-35). These characteristics render IGL-1 suitable for abdominal organ preservation. ${ }^{7-11}$ However, the mechanisms by which IGL-1 protects against ischemia reperfusion (I/R) injury and maintains cell viability are unclear. Accumulating evidence suggested perturbations at the endoplasmic reticulum $(E R)$ as novel subcellular effectors, possibly involved in the promotion of cell death during cold organ preservation. ${ }^{12-14}$ Indeed, I/R stimulates a large increase in intracellular $\mathrm{Ca}^{2+}$ concentration resulting from a depletion of $\mathrm{Ca}^{2+}$ from the ER lumen. ${ }^{15}$ In turn, this mobilization of $\mathrm{Ca}^{2+}$ modulates mitochondrial $\mathrm{Ca}^{2+}$ responses and hepatocyte susceptibility to apoptosis, which are manifested by the release of cytochrome $c$ and caspase- 9 activation. Moreover, once the ER homeostasis is perturbed, newly synthesized unfolded proteins accumulate in the organelle, resulting in ER stress. ${ }^{16,17}$ To cope with accumulated unfolded proteins, mammalian cells trigger a specific adaptative response called the unfolded protein response (UPR). ${ }^{12,16,17}$

The UPR has three branches: inositol-requiring enzyme 1 (IRE1), PKR-like ER kinase (PERK) and activating transcription factor (ATF6). These proteins are normally held in inactive states in ER membranes by binding to intra-ER chaperones, particularly the 78-kD glucose-regulated/binding immunoglobulin protein (GRP78). In response to stimuli that divert ER chaperones to misfolded proteins, IRE1, PERK and ATF6

\footnotetext{
${ }^{1}$ Experimental Hepatic Ischemia-Reperfusion Unit, IIBB-CSIC Barcelona, Barcelona, Spain; ${ }^{2}$ INSERM UMR-S 769, LabEx LERMIT, Université de Paris Sud, Faculté de Pharmacie, Châtenay-Malabry 92296, France and ${ }^{3}$ Department of Pharmacy, Laboratory of Human Physiology, University of Monastir, Avenue Ali Bourguiba - Skanes, BP 56, Monastir 5000, Tunisia

*Corresponding author: C Brenner, INSERM UMR-S 769, LabEx LERMIT, Université de Paris Sud, Faculté de Pharmacie, Châtenay-Malabry 92296, France.

Tel: + 133 6609977; Fax: + 133 139254572; E-mail: catherine.brenner-jan@u-psud.fr

${ }^{4}$ These authors contributed equally to this work.

Keywords: unfolded protein response; endoplasmic reticulum stress; oxidative stress; liver transplantation; ischemia injury

Abbreviations: I/R, ischemia reperfusion; UPR, unfolded protein response; ER, endoplasmic reticulum; IGL-1 solution, Institute of Georges Lopez-1 solution; UW, University of Wisconsin; IRE1, inositol-requiring enzyme; GRP78, glucose-regulated/binding immunoglobulin protein; XBP-1, X-box-binding protein; TRAF2, tumor necrosis factor-associated factor 2; elF2 $\alpha$, eukaryotic translation initiation factor 2; HES, hydroxyethyl starch; PEG, polyethylene glycol; PERK, PKR-like ER kinase; ATF, activating transcription factor; TNF, tumor necrosis factor; JNK, c-Jun N-terminal kinase; LDH, lactate dehydrogenase; AST, aspartate amino transferase; ALT, alanine amino transferase; GLDH, glutamate dehydrogenase

Received 25.7.11; revised 29.12.11; accepted 30.1.12; Edited by M Federici
} 
initiate signal transduction processes. These events promote the expression of genes required to fold newly synthesized proteins and to degrade the unfolded proteins. Moreover, homeostasis and normal ER function are restored. However, when injury is excessive, the same ER stress signal transduction pathways can also induce cell death. ${ }^{12,16,17}$ The first of the three branches of the UPR includes IRE $1 \alpha$, which, once activated, induces the unconventional splicing of the mRNA encoding X-box-binding protein 1 (XBP-1). The cytosolic domain of activated IRE $1 \alpha$ binds the tumor necrosis factor (TNF)-associated factor 2 (TRAF2), and triggers the activation of the c-Jun N-terminal kinase (JNK), MAPK p38 and caspase-12. ${ }^{16,18}$ The second branch is mediated by PERK, which phosphorylates $\operatorname{Ser}^{21}$ of the $\alpha$ subunit of eukaryotic translation initiation factor 2 (elF2 $\alpha$ ). elF2 $\alpha$ phosphorylation induces translation of a basic-region leucine zipper transcription factor ATF4 and subsequent expression of the ATF4 target genes, C/EBP homologous protein-10 (CHOP). ${ }^{12,16,19}$ The third branch is mediated by the basicregion leucine zipper transcription factor ATF6, which is activated by regulated intramembrane proteolysis. ${ }^{12,16,20}$

We report the results of an experimental study that aimed to evaluate the involvement of ER stress following liver transplantation and to assess whether IGL-1 solution is more protective than UW solution against hepatic l/R injury through the attenuation of ER stress. The aim of the present study was not to distinguish between a specific and beneficial effect of $\mathrm{Na}^{+}$or PEG, or a synergistic effect, but to combine the cationic inversion and HES replacement by PEG to obtain the maximal protection of liver graft against ER stress induced by cold ischemia-reperfusion injury.

\section{Results}

Induction of UPR after liver transplantation. In order to determine whether the UPR was activated upon I/R injury associated with liver transplantation, we assessed the expression of the following three mRNAs known to be specifically induced by ER stress: GRP78, an ER chaperone, as well as CHOP and GADD34, two genes involved in growth arrest and DNA damage. We used semi-quantitative real-time PCR (Figure 1a) and western blot (Figure 1b). GRP78, CHOP and GADD34 mRNA (Figure 1a) and protein expression (Figure 1b) were induced after liver transplantation compared with the Sham group. However,

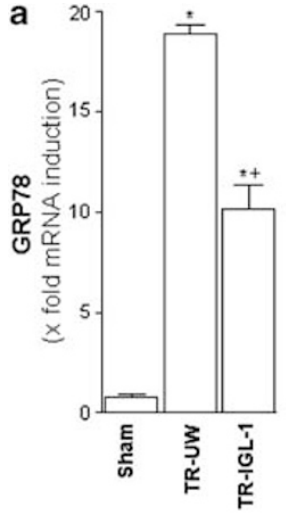

b
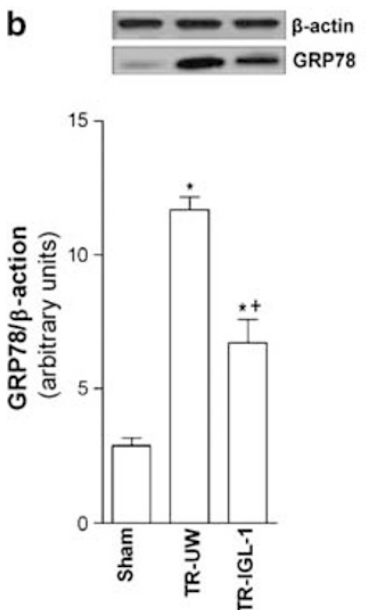
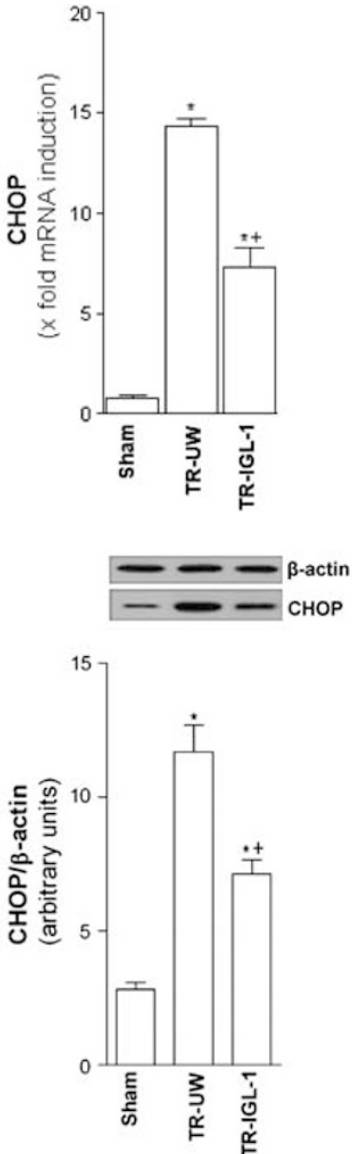
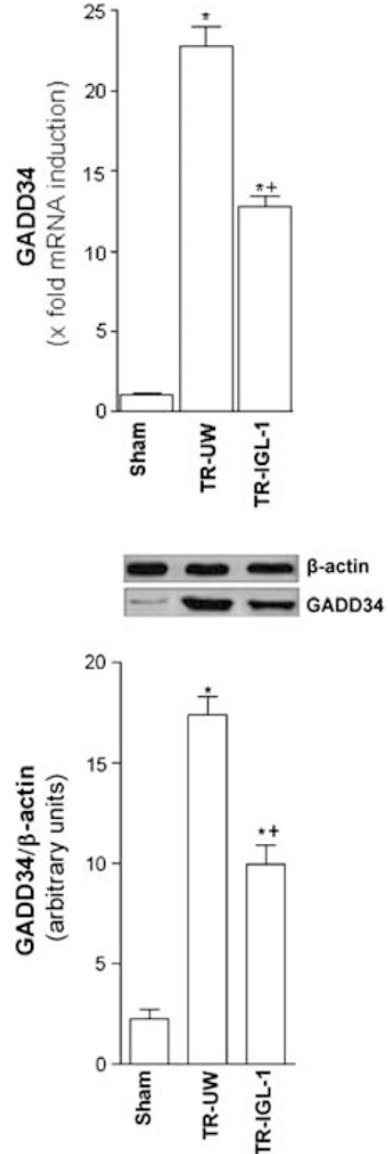

Figure 1 UPR induction after liver transplantation. (a) mRNA expressions and (b) protein level of GRP78, CHOP and GADD34 in grafts preserved in UW solution (TR-UW) or IGL-1 solution (TR-IGL-1). For GRP78, CHOP and GADD34 mRNA expressions in the liver, PCR fluorescent signals for GRP78, CHOP and GADD34 were standardized to PCR fluorescent signals obtained from an endogenous reference ( $\beta$-actin). Comparative and relative quantifications of these gene products normalized to $\beta$-actin and the Sham group were calculated by the $2^{-\Delta \Delta C T}$ method. For GRP78, CHOP and GADD34 protein levels in the liver, the upper panels show one representative blot of five independent experiments and the lower panels show densitometric evaluation of the independent western blot. $N=5$, error bars denote $\pm S$.E.M. ${ }^{\star} P<0.05$ versus Sham; ${ }^{+} P<0.05$ versus TR-UW 
a
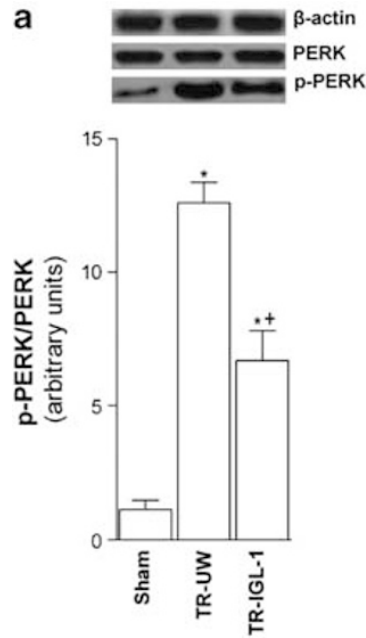

b
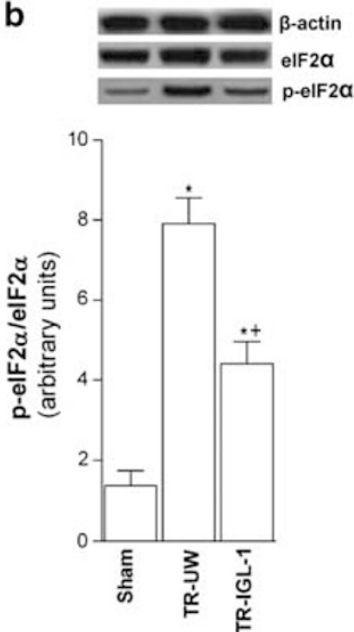

C
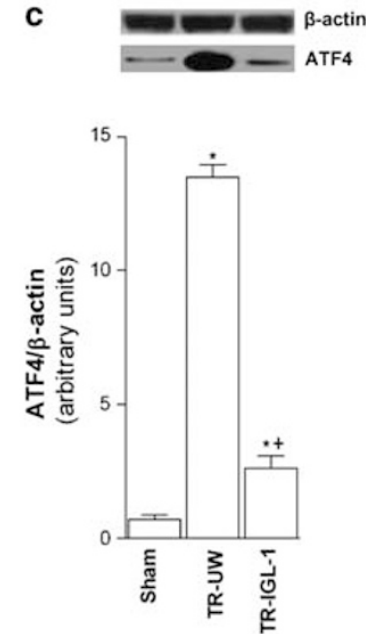

Figure 2 PERK pathway activation after liver transplantation. (a) Protein level of total and phospho-PERK, (b) total and phospho-elF2 $\alpha$ and (c) ATF4 in livers grafts preserved in UW solution (TR-UW) or IGL-1 solution (TR-IGL-1). The upper panels show one representative blot of five independent experiments and the lower panels show densitometric evaluation of the independent western blot. $N=5$, error bars denote \pm S.E.M. ${ }^{\star} P<0.05$ versus Sham; ${ }^{+} P<0.05$ versus TR-UW

\section{a}
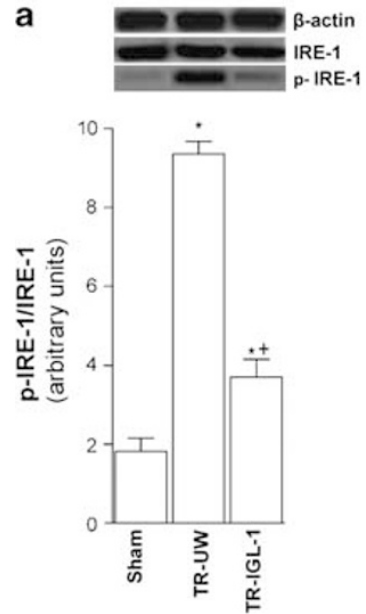

b
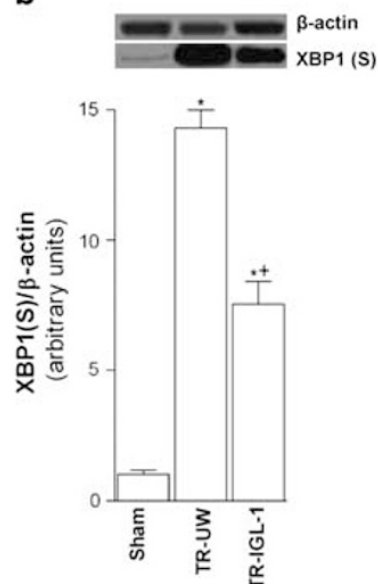

C
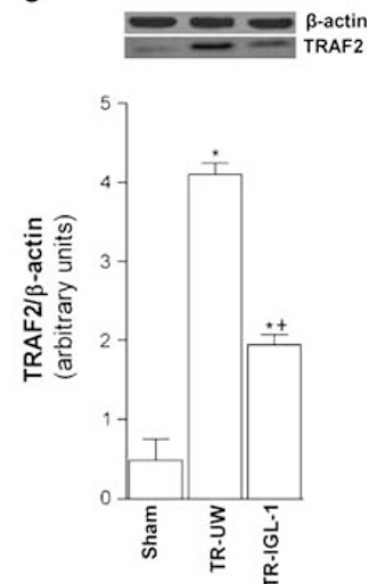

Figure 3 IRE-1 pathway activation after liver transplantation. (a) Protein level of total and phospho-IRE1, (b) XBP1 and (c) TRAF2 in livers grafts preserved in UW solution (TR-UW) or IGL-1 solution (TR-IGL-1). The upper panels show one representative blot of five independent experiments and the lower panels show densitometric evaluation of the independent western blot. $N=5$, error bars denote \pm S.E.M. ${ }^{*} P<0.05$ versus Sham; ${ }^{+} P<0.05$ versus TR-UW

GRP78, CHOP and GADD34 mRNA and protein expression were lower when IGL-1 solution was used than when UW solution was used (Figures 1a and b). As the induction of GRP78, CHOP and GADD34 are indicative of the activation of the UPR, we examined which of the three branches of the UPR (PERK, IRE-1 and ATF6) were activated after liver transplantation (Figures 2, 3 and 4).

PERK To evaluate a specific activation of PERK pathway following liver transplantation, we assessed hepatic expression levels of phospho PERK, and phospho elF2 $\alpha$ and ATF4 activation by western blot using specific antibodies. Levels of phospho PERK increased (Figure 2a), concomitantly with elF2 $\alpha$ phosphorylation (Figure 2b) and ATF4 accumulation (Figure 2c). However, the activation of PERK, elF2 $\alpha$ and ATF4 were significantly lower for IGL-1 solution used than for UW solution (Figure 2).
$I R E-1$ To assess a specific activation of IRE-1 pathway, we examined hepatic phospho-IRE-1 and XBP-1s protein expression. I/R associated with liver transplantation increased phospho-IRE-1 (Figure 3a), and XBP-1s (Figure 3b), protein levels when compared with the Sham group. However, protein phospho-IRE-1 and XBP-1(S) levels were lower in the TR-IGL-1 group than in the TR-UW group (Figures $3 a$ and $b$ ). Activated IRE-1 has been also shown to be upstream of the activation of TRAF2. Similar to the results of XBP-1, $\mathrm{I} / \mathrm{R}$ increased TRAF2 protein levels (Figure $3 \mathrm{c}$ ), but the use of IGL-1 significantly reduced TRAF2 (Figure 3c). This indicates that IGL-1 solution reduced the IRE-1 pathway activity after liver transplantation.

ATF6 To evaluate the activation of ATF6 pathway following liver transplantation, we analyzed the effect of $\mathrm{I} / \mathrm{R}$ on hepatic p50ATF6 $\alpha$ and p50ATF6 $\beta$ protein expression. Liver 

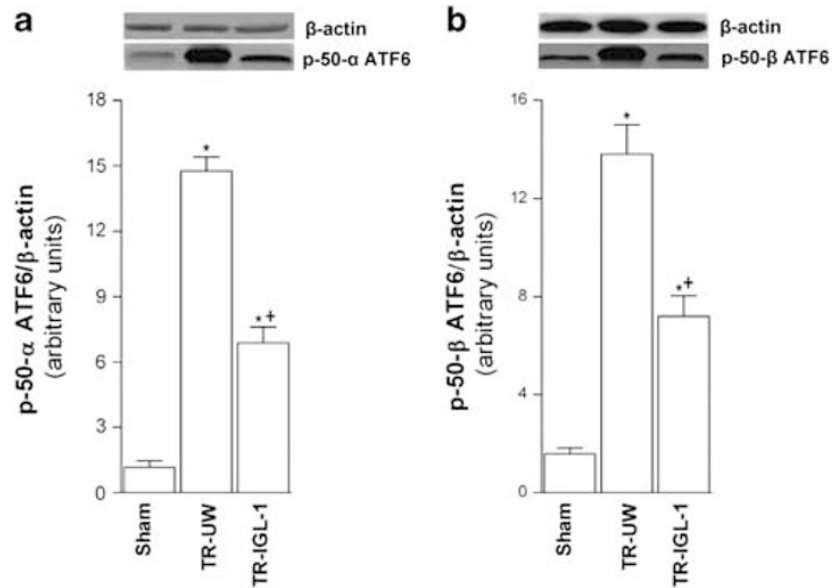

Figure 4 ATF6 pathway activation after liver transplantation. (a) Protein level of p50ATF6 $\alpha$ and (b) p50ATF6 $\alpha$ in livers grafts preserved in UW solution (TR-UW) or IGL-1 solution (TR-IGL-1). The upper panels show one representative blot of five independent experiments and the lower panels show densitometric evaluation of the independent western blot. $N=5$, error bars denote \pm S.E.M. ${ }^{*} P<0.05$ versus Sham; ${ }^{+} P<0.05$ versus TR-UW transplantation increased the expression of p50ATF6 $\alpha$ (Figure 4a) and p50ATF6 $\beta$ (Figure 4b) compared with the Sham group. However, IGL-1 solution significantly reduced these expression levels compared with UW solution (Figures $4 \mathrm{a}$ and $\mathrm{b}$ ).

MAPK kinase signaling To determine whether MAPK kinase signaling pathway was activated after liver transplantation, we examined the activation of JNK1/2 and p38 MAPK. Phosphorylation levels of JNK1/2 (Figure 5a) and p38 MAPK (Figure $5 \mathrm{~b}$ ) were significantly higher after liver transplantation compared with the Sham group. IGL-1 solution significantly reduced the phosphorylated levels of JNK1/2 and p38 MAPK compared with UW solution (Figures $5 \mathrm{a}$ and b).

Inflammatory response and hepatic injury. Owing to the association of inflammatory response and hepatocellular damage in liver transplantation with ER stress response, we examined the expression of inflammatory mediators (TNF $\alpha$, IL1 $\beta$ and IL6) and alanine amino transferase (ALT), aspartate amino transferase (AST) and lactate a
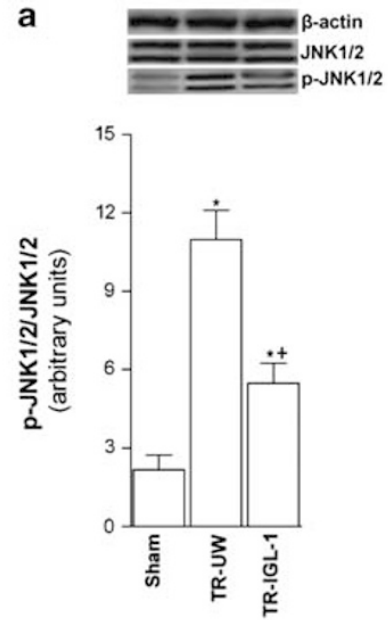

b
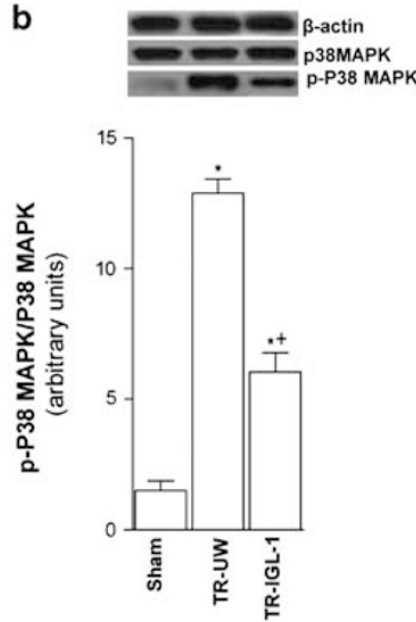
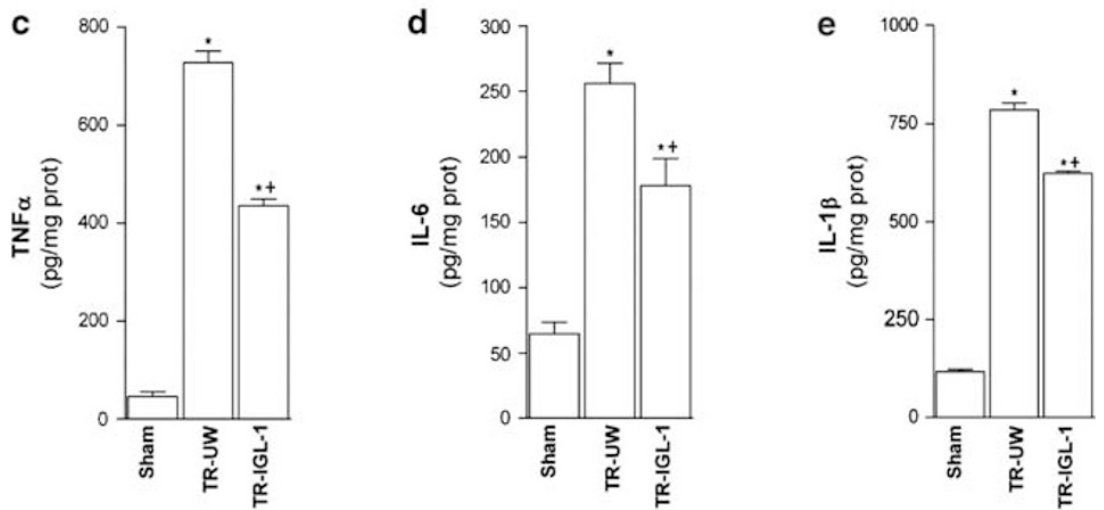

Figure 5 JNK and P38 MAPK activation and inflammatory response after liver transplantation. (a) Protein levels of total and phospho p-JNK and (b) total and phosphoP38MAPK in liver grafts preserved in UW solution (TR-UW) or IGL-1 solution (TR-IGL-1). The upper panels show one representative blot of five independent experiments and the lower panels show densitometric evaluation of the independent western blot. $N=5$, error bars denote \pm S.E.M. ${ }^{*} P<0.05$ versus Sham; ${ }^{+} P<0.05$ versus TR-UW. (c) Hepatic level of TNF $\alpha$, (d) IL6 and (e) IL1 $\beta$ in grafts preserved in UW solution (TR-UW) or IGL-1 solution (TR-IGL-1). $N=6$, error bars denote $\pm S$.E.M. ${ }^{*} P<0.05$ versus Sham; ${ }^{+} P<0.05$ versus TR-UW 

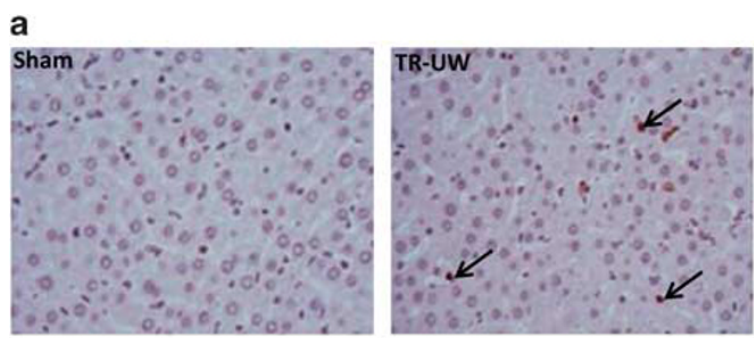

b

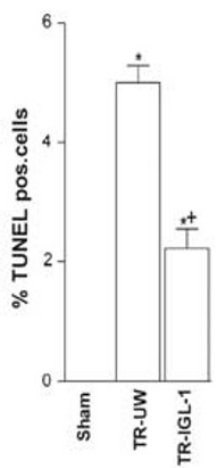

C

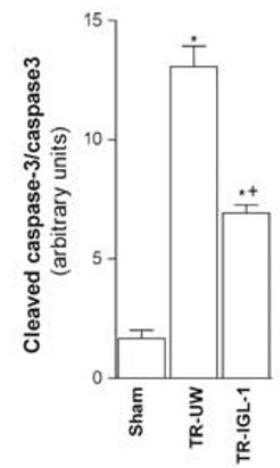

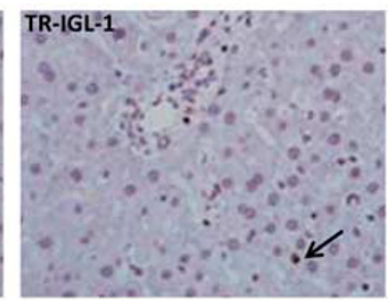

d
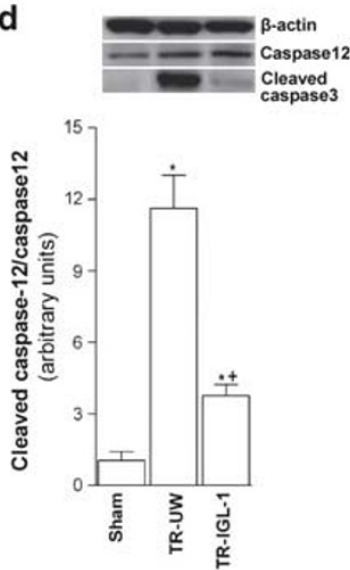

Figure 6 Cell death after liver transplantation. (a) TUNEL staining of the livers (original magnification $\times 400$ ). Any hepatocytes stained TUNEL positive in the Sham group. Some hepatocytes stained TUNEL positive in the TR-UW group (black arrowhead). TUNEL-positive hepatocytes were significantly decreased in group TR-IGL-1. (b) Percentage of TUNEL-positive hepatocyte. (c) Protein levels of cleaved caspase-3 and (d) caspase-12 expression in grafts preserved in UW solution (TR-UW) or IGL-1 solution (TR-IGL-1). The upper panels show one representative blot of five independent experiments and the lower panels show densitometric evaluation of the independent western blot. $N=5$, error bars denote \pm S.E.M. ${ }^{*} P<0.05$ versus Sham; ${ }^{+} P<0.05$ versus TR-UW

Table 1 Serum levels of ALT, AST and LDH in grafts preserved in UW solution (TR-UW) or IGL-1 solution (TR-IGL-1)

\begin{tabular}{lccr}
\hline & \multicolumn{3}{c}{ Experimental groups } \\
\cline { 2 - 4 } & Sham & \multicolumn{1}{c}{ TR-UW } & \multicolumn{1}{c}{ TR-IGL-1 } \\
\hline ALT (U/I) & $62 \pm 4$ & $602 \pm 44^{*}$ & $280 \pm 32^{\star+}$ \\
AST (U/I) & $76 \pm 3$ & $1120 \pm 122^{*}$ & $810 \pm 79^{\star+}$ \\
LDH (U/I) & $982 \pm 74$ & $3662 \pm 104^{*}$ & $2062 \pm 54^{\star+}$ \\
\hline
\end{tabular}

$N=6,{ }^{*} P<0.05$ versus Sham; ${ }^{+} P<0.05$ versus TR-UW.

dehydrogenase (LDH). TNF $\alpha$ (Figure 5c), IL6 (Figure 5d) and IL1 $\beta$ (Figure 5e) levels were higher after liver transplantation compared with the Sham group. Nevertheless, IGL-1 solution significantly decreased these levels compared with UW solution (Figures $5 \mathrm{c}$ and $\mathrm{d}$ and $6 \mathrm{e}$ ). We report here increased hepatocellular damage after liver transplantation evidenced by increases in transaminases (ALT and AST) and LDH compared with the Sham group (Table 1). However, these increases were significantly offset by IGL-1 as preservation solution compared with the liver preserved in UW solution (Table 1).

Hepatic cell death. Excessive stress to the ER during organ preservation compromises cellular homeostasis indirectly. Here, we examined the apoptosis evaluated by percentage of TUNEL-positive hepatocytes, and by protein expression of cleaved caspase-3 and caspase-12. I/R associated with liver transplantation increased the percentage of TUNEL-positive cell (Figures 6a and b), as well as cleaved caspase-3 (Figure 6c) and caspase-12 (Figure 6d) expressions when compared with the Sham group. Decreased TUNEL-positive cells, cleaved caspase-3 and caspase-12 expression were observed when IGL-1 solution was used as the preservation solution compared with UW solution.

Mitochondrial damage. Considering the potential relationship between ER stress and mitochondrial damage, we examined mitochondrial damage by measurement of plasma glutamate dehydrogenase (GLDH) activity and cytosolic cleaved caspase-9, and mitochondrial and cytosolic cytochrome $c$ activities following liver transplantation. Liver transplantation induced activation of GLDH (Figure 7a), cleaved caspase-9 (Figure 7b) and cytochrome $c$ (Figure 7c) compared with values in the Sham group. However, IGL-1 reduced this activation compared with liver UW solution. This was evidenced by significant reduction in GLDH activity (Figure 7a) and protein expression of cleaved caspase-9 (Figure 7b) and cytosolic cytochrome $c$ (Figure 7c) in the TR-IGL-1 group compared with the TR-UW group.

\section{Discussion}

The quality of organ preservation during ischemic storage may have a significant impact on liver allograft outcome after 
a

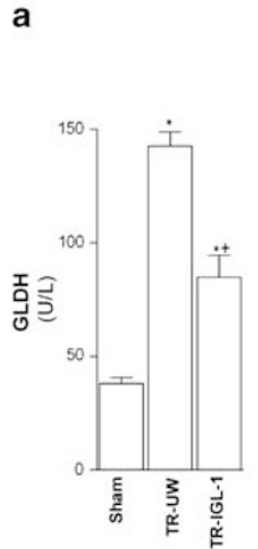

b
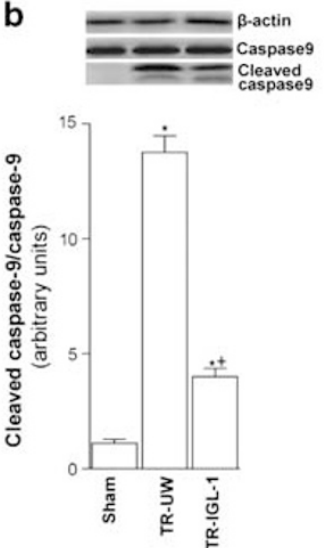

c
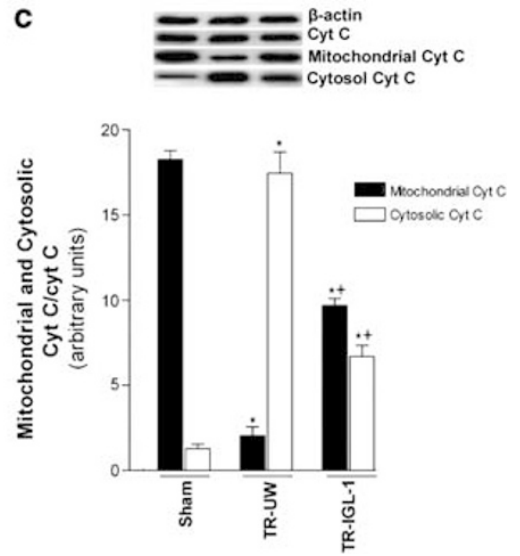

Figure 7 Mitochondrial damage after liver transplantation. (a) GLDH plasma level and (b) protein level of cleaved caspase-9 and (c) cytochrome $c$ expression in grafts preserved in UW solution (TR-UW) or IGL-1 solution (TR-IGL-1). The upper panels show one representative blot of five independent experiments and the lower panels show densitometric evaluation of the independent western blot. $N=5$, error bars denote \pm S.E.M. ${ }^{\star} P<0.05$ versus Sham; ${ }^{+} P<0.05$ versus TR-UW

transplantation and may affect the risk of long-term complications. Thus, any improvement in the preservation of liver grafts may increase the number of viable donor livers available for transplantation.

Consistent with previous studies and that from our laboratory, ${ }^{7,8,11} \mathrm{IGL}-1$ solution, based on the use of low $\mathrm{K}^{+}$ high $\mathrm{Na}^{+}$concentrations and replacement of HES by PEG, while retaining all the advantages of original UW solution, improved hepatic I/R injury. The mechanism by which IGL-1 prevents microcirculatory alteration and cell swelling, and maintains cell viability remains to be determined. In the present study, we investigate the involvement of ER stress in liver I/R injury associated with liver transplantation, comparing cold storage in UW solution and IGL-1 solution. We report the accentuation of ER response following liver transplantation. The use of IGL-1 as the preservation solution significantly reduced ER stress. This reduction was associated with a decrease in inflammatory response, mitochondrial damage and hepatocellular injury. Indeed, we identified the activation of ER stress signaling pathways that are affected at transcriptional, post-transcriptional and post-translational levels. We showed induction of three mRNAs (GRP78, CHOP and GADD34) known to be specifically induced upon ER stress. A significant attenuation of the GRP78, CHOP and GADD34 mRNA and protein expression were observed when IGL-1 solution was used as the preservation solution. Additionally, the data of the present study show the activation of all three UPR arms: PERK, ATF6 and IRE1, and their downstream targets, evidenced by the induction of elF2 $\alpha$, TRAF2, XBP-1(S) and ATF4 after liver transplantation compared with the Sham group. In line with these results, activation of ER stress markers has been observed in tissue biopsies from human allograft after reperfusion. Indeed, Emadali et al. ${ }^{21}$ demonstrate an upregulation of several ER stress response chaperones in the liver during reperfusion, supporting the putative impact of alterations at the level of the ER during I/R-induced cell damage. However, here a significant reduction of activity of three UPR arms and their downstream targets was observed when livers were preserved in IGL-1 solution. These results confirm the protective effect of IGL-1 solution on the ER stress induced after liver transplantation. It has been proposed that activation of the IRE-1 pathway regulates pro-apoptotic responses by activation of stress kinase JNK and mitogen-activated protein kinase. $^{22}$ The data of the present study reflected changes in protein phosphorylation indicating activation of JNK1/2 and P38 MAPK after liver transplantation. Our results show that the use of IGL-1 solution significantly reduces the expression of phospho JNK1/2 and P38 MAPK compared with UW solution. Taken together, these data suggest that IGL-1 protects the liver against I/R-induced apoptosis through the attenuation of ER stress, which in turn may lead to inhibition of the JNK1/2 and P38 MAPK pathways. As it has also been shown that MAPK activation can be triggered by reactive oxygen species production, ${ }^{23}$ we cannot rule out that the inhibition of these kinases observed when IGL-1 solution was used is independent of the ER pathway attenuation. This protective role of IGL-1 solution in oxidative stress may be directly related with the presence of PEG and $\mathrm{Na}^{+} / \mathrm{K}^{+}$ inversion in the solution, and its antioxidant properties previously demonstrated in perfused rat kidney ${ }^{24}$ and in isolated cold-stored rat hepatocytes. ${ }^{25,26}$ Nevertheless, the mechanism by which specific PEG-35 in IGL-1 solution acts as an antioxidant molecule is unknown. On the other hand, it is well known that the UPR has been linked to several inflammatory response pathways in many cellular models and diseases. ${ }^{12,27}$ Moreover, caspase activation links CHOP to both pro-inflammatory mediators and apoptosis, and ER stress may activate the mitochondrial apoptosis pathway. ${ }^{12,28-30}$ Our results demonstrate that IGL-1 significantly decreases the inflammatory response, reflected by values of TNF $\alpha, \operatorname{IL} 1 \beta$ and IL6 expression, compared with livers preserved in UW solution. This was associated with an important reduction of hepatic parenchymal enzyme release, evidenced here by decreased ALT, AST and LDH levels. This confirms the advantage of IGL-1 solution for protecting against hepatocellular injury associated with liver transplantation. Apoptosis is considered a hallmark of I/R-induced graft damage. The proposed pathways of this apoptosis execution after I/R include cascade-like proteolytic cleavage 
activation of cysteine-like proteases, such as caspase-3 and caspase-12, a pro-apoptotic factor especially related to ER stress. Our results show slightly lower cleaved caspase-3 activity and strongly reduced caspase- 12 expression, as well as reduced cell death when livers were preserved in IGL-1 solution. This attenuation of cell death and inflammatory response by IGL-1 solution was associated with an improvement of mitochondrial integrity, reflected by decreased GLDH activity, cleaved caspase-9 and cytosolic cytochrome $c$ expression. This correlates with a strict relation between ER stress and mitochondrial damage as previously demonstrated. $^{12,28,30,31}$ The mechanisms by which IGL-1 solution protects from ER stress and maintains cell viability remain unclear. Based on our previous work, ${ }^{8}$ one of the current hypotheses to explain these protective effects is related with the higher capacity of IGL-1 solution to induce constitutive nitric oxide, which is considered vital for the induction of antioxidant defense genes and those involved in ER protein folding. ${ }^{32}$ Other potential benefits of IGL-1 solution related to PEG and lower potassium concentration, such as the antiaggregating effect of $P E G,{ }^{5}$ improved microcirculation, as well as an 'immuno-camouflaging' effect of $P E G,{ }^{33}$ which protects against the initial inflammatory response, ${ }^{34}$ should not be ruled out.

The exact relationship of ER stress-mediated cell death and cold $\mathrm{I} / \mathrm{R}$ injury in liver transplantation will require further definition, but results presented here implicate ER stress on a broad scale as an important factor in this injury. We suggest that ER stress is an important potential target of IGL-1 to improve liver transplantation. However, time-course analysis of ER stress after liver transplantation might lead to a better understanding of the implication of ER stress in liver transplantation before these experimental results are confirmed in a clinical study. In conclusion, we delineate a role of ER during preservation/reperfusion of pre-damaged liver grafts, which is aggravated by the use of UW solution and attenuated by the use of IGL-1 solution. Thus, IGL-1 preservation solution may be a useful means to circumvent excessive endoplasmic stress reactions, thereby combining with other advantages of this liquid compared with standard UW solution. It may thus be a valuable alternative to UW to prevent I/R injury associated with liver transplantation and it may optimize graft quality.

\section{Materials and Methods}

Experimental animals. Male Sprague-Dawley rats weighing $250-300 \mathrm{~g}$ were used as donors and recipients. All animals were anesthetized with isofluorane. Research procedures complied with the European Union regulations for animal experiments (EC-guideline 86/609/CEE).

Experimental groups. All animals were randomly distributed into three groups as described below. The groups consisted of: (i) a sham $(n=8)$ group of animals subjected to anesthesia, transverse laparotomy and silk ligatures in the right suprarenal vein, diaphragmatic vein and hepatic artery; (ii) transplantation group using UW solution (TR-UW) ( $n=16$, eight transplantations), in which donor livers were flushed and preserved with cold $\left(4^{\circ} \mathrm{C}\right)$ UW solution (Viaspan, Madrid, Spain) for $8 \mathrm{~h}^{35,36} \mathrm{~A}$ standard orthotopic liver transplantation was performed according to Kamada's cuff technique, without hepatic artery construction. ${ }^{37}$ The anhepatic phase lasted $17-20 \mathrm{~min}$, as described previously. ${ }^{35,36}$ Liver transplantation with grafts undergoing $8 \mathrm{~h}$ of cold ischemia is associated with high survival. ${ }^{36,38}$ In the third group (TR-IGL-1) (iii), the same surgical procedures as described for group (ii) were carried out, but livers were flushed and preserved in cold $\left(4^{\circ} \mathrm{C}\right)$ IGL-1 solution (Institute Georges Lopez-1 solution, Lyon, France). At 24 hours after transplantation, serum was obtained and aliquots of the liver were flash frozen for RNA and protein extraction.

Reverse transcription and real-time PCR. Quantitative real-time PCR analysis was performed using the Assays-on-Demand TaqMan probe ((Rn 00565250-m1 for GRP78) (Rn00492098_g1 for CHOP) and (Rn00591894_m1 for GADD34)) (Applied Biosystems, Foster City, CA, USA). The TaqMan gene expression assay was performed according to the manufacturer's protocol (Applied Biosystems).

Western blotting. Liver tissue was homogenized as previously described. ${ }^{28,39,40}$ Total liver lysates were then used to quantify GRP78, CHOP, GADD34, ATF4, ATF $_{\alpha}$, ATF $_{\beta}, \mathrm{XBP} 1(\mathrm{~S})$, TRAF2, $\beta$-actin, cleaved caspase-3, caspase-12, total and phospho-PERK, total and phospho-IRE-1, total and phospho-elF2 ${ }_{\alpha}$, total and phospho-p38 MAPK and total and phospho-JNK1/2 by western blot. Cytosolic fractions were used to quantify cleaved caspase- 9 and cytochrome $c$ by western blot. Mitochondrial fractions were used to quantify mitochondrial cytochrome $c$ by western blot. Proteins were separated by sodium dodecyl sulfate PAGE and transferred to polyvinylidene fluoride membranes. Membranes were immunoblotted with antibodies directed against GRP78, CHOP, GADD34, ATF4, p50-ATF6 ${ }_{\alpha}$, p50$\mathrm{ATF}_{\beta}, \mathrm{XBP1}(\mathrm{S})$, caspase-12, $\beta$-actin, total and phospho-PERK and total and phospho-elF2 $\alpha$ (Santa Cruz Biotechnology, Santa Cruz, CA, USA), TRAF2, cytochrome $c$, cleaved caspase-3 and cleaved caspase-9, total and phospho-p38 MAPK and total and phospho-JNK1/2 (Cell Signaling Technology Inc., Beverly, MA, USA) and total and phospho-IRE-1 (Thermo Fisher Scientific, Illkirch, France). The bands were visualized using an enhanced chemiluminescence kit (Bio-Rad Laboratories, Hercules, CA, USA). The values were obtained by densitometric scanning and the Quantity One software program (Bio-Rad Laboratories) The scanning values for GRP78, CHOP, GADD34, ATF4, TRAF2, p50-ATF6 $\alpha_{\alpha}$, p50$\mathrm{ATF}_{\beta}$, and XBP1(S) were divided by scanning values of $\beta$-actin, and those for phosphorylated PERK, elF2 ${ }_{\alpha}$, IRE-1, JNK1/2, P38MAPK, and cytosolic and mitochondrial cytochrome $c$ were divided by the total PERK, elF2 $\alpha_{\alpha}, \mathrm{IRE}-1, \mathrm{JNK} 1 / 2$, p38MAPK, and cytosolic and mitochondrial cytochrome $c$, respectively. Cleaved caspase- $3,-9$ and -12 were divided by the full-length fragment of each protein, respectively. All signals were normalized to the corresponding Ponceau S. ${ }^{7,8}$

Determination of proinflammatory cytokines. Hepatic rat cytokines (TNF $\alpha$, IL- 6 and IL-1 $\beta$ ) in the liver were assayed according to the ELISA protocol given by the supplier (Invitrogen Corporation, Alcobendas, Spain).

Biochemical determinations. Plasma levels of AST, ALT and LDH were determined using commercial kits from Boehringer Mannheim (Munich, Germany).

Determination of glutamate dehydrogenase activity. GLDH is a mitochondrial enzyme, which was used as an indirect measure of mitochondrial damage. GLDH activity was measured in plasma samples, as described elsewhere. ${ }^{40}$

TUNEL assay. DNA fragmentation was determined using a TUNEL assay in deparaffinized liver samples, as described elsewhere. ${ }^{28,41}$ TUNEL-positive nuclei were counted in 10 random high-power fields ( $\times 40$ objectives).

Statistics. Data are expressed as means and S.E.'s. Differences between groups were evaluated with the Student $t$-test followed by the Mann-Whitney U-test. $P<0.05$ was considered significant.

\section{Conflict of Interest}

The authors declare no conflict of interest.

Acknowledgements. We are grateful to Robin Rycroft at the language advisory service of the University of Barcelona for revising the English text. This work was supported by The Ministry of Health and Consumption (PI 081988), CIBER-ehd, Carlos III Institute, Madrid, Spain; Ministry of Foreign Affairs and International Cooperation (A/020255/08 and A/02987/09).

1. Bartels-Stringer M, Kramers C, Wetzels JF, Russel FG, Groot H, Rauen U. Hypothermia causes a marked injury to rat proximal tubular cells that is aggravated by all currently used preservation solutions. Cryobiology 2003; 47: 82-91. 
2. Belzer FO, Southard JH. Principles of solid-organ preservation by cold storage. Transplantation 1988; 45: 673-676.

3. Mor E, Klintmalm GB, Gonwa TA, Solomon H, Holman MJ, Gibbs JF et al. The use of marginal donors for liver transplantation. A retrospective study of 365 liver donors. Transplantation 1992; 53: 383-386.

4. Todo S, Nery J, Yanaga K, Podesta L, Gordon RD, Starzl TE. Extended preservation of human liver grafts with UW solution. JAMA 1989; 261: 711-714.

5. Mosbah IB, Franco-Gou R, Abdennebi HB, Hernandez R, Escolar G, Saidane D et al. Effects of polyethylene glycol and hydroxyethyl starch in University of Wisconsin preservation solution on human red blood cell aggregation and viscosity. Transplant Proc 2006; 38: 1229-1235

6. van der Plaats A, 't Hart NA, Morariu AM, Verkerke GJ, Leuvenink HG, Ploeg RJ et al. Effect of University of Wisconsin organ-preservation solution on haemorheology. Transpl Int 2004; 17: 227-233.

7. Zaouali MA, Ben Mosbah I, Boncompagni E, Ben Abdennebi H, Mitjavila MT, Bartrons R et al. Hypoxia inducible factor-1alpha accumulation in steatotic liver preservation: role of nitric oxide. World J Gastroenterol 2010; 16: 3499-3509.

8. Ben Mosbah I, Roselló-Catafau J, Franco-Gou R, Abdennebi HB, Saidane D, RamellaVirieux $S$ et al. Preservation of steatotic livers in IGL-1 solution. Liver Transpl 2006; 12: 1215-1223.

9. Badet L, Ben Abdennebi H, Petruzzo P, McGregor B, Espa M, Hadj-Aissa A et al. Effect of IGL-1, a new preservation solution, on kidney grafts (a pre-clinical study). Transpl Int 2005; 17: 815-821.

10. Maathuis MH, Ottens PJ, van Goor H, Zwaagstra JJ, Wiersema-Buist J, Schuurs TA et al. Static cold storage preservation of ischemically damaged kidneys. A comparison between IGL-1 and UW solution. Transpl Int 2008; 21: 473-482.

11. Ben Abdennebi H, Elrassi Z, Scoazec JY, Steghens JP, Ramella-Virieux S, Boillot 0 . Evaluation of IGL-1 preservation solution using an orthotopic liver transplantation model. World J Gastroenterol 2006; 12: 5326-5330.

12. Peralta $C$, Brenner $C$. Endoplasmic reticulum stress inhibition enhances liver tolerance to ischemia/reperfusion. Curr Med Chem 2011; 18: 2016-2024.

13. Manekeller S, Schuppius A, Stegemann J, Hirner A, Minor T. Role of perfusion medium, oxygen and rheology for endoplasmic reticulum stress-induced cell death after hypothermic machine preservation of the liver. Transpl Int 2008; 21: 169-177.

14. Minor T, Manekeller S, Sioutis M, Dombrowski F. Endoplasmic and vascular surface activation during organ preservation: refining upon the benefits of machine perfusion. $A m \mathrm{~J}$ Transplant 2006; 6: 1355-1366.

15. Pallet N, Fougeray S, Beaune P, Legendre C, Thervet E, Anglicheau D. Endoplasmic reticulum stress: an unrecognized actor in solid organ transplantation. Transplantation 2009; 88: 605-613.

16. Xu C, Bailly-Maitre B, Reed JC. Endoplasmic reticulum stress: cell life and death decisions. J Clin Invest 2005; 115: 2656-2664.

17. Ozcan U, Yilmaz E, Ozcan L, Furuhashi M, Vaillancourt E, Smith RO et al. Chemical chaperones reduce ER stress and restore glucose homeostasis in a mouse model of type 2 diabetes. Science 2006; 313: 1137-1140.

18. Yoneda T, Imaizumi K, Oono K, Yui D, Gomi F, Katayama T et al. Activation of caspase-12, an endoplastic reticulum (ER) resident caspase, through tumor necrosis factor receptorassociated factor 2-dependent mechanism in response to the ER stress. J Biol Chem 2001; 276: $13935-13940$

19. Rao RV, Ellerby HM, Bredesen DE. Coupling endoplasmic reticulum stress to the cell death program. Cell Death Differ 2004; 11: 372-380.

20. Ye J, Rawson RB, Komuro R, Chen X, Dave UP, Prywes R et al. ER stress induces cleavage of membrane-bound ATF6 by the same proteases that process SREBPs. Mol Cell 2000; 6: 1355-1364.

21. Emadali A, Nguyên DT, Rochon C, Tzimas GN, Metrakos PP, Chevet E. Distinct endoplasmic reticulum stress responses are triggered during human liver transplantation. J Pathol 2005; 207: 111-118.

22. Nguyên DT, Kebache S, Fazel A, Wong HN, Jenna S, Emadali A et al. Nck-dependent activation of extracellular signal-regulated kinase-1 and regulation of cell survival during endoplasmic reticulum stress. Mol Biol Cell 2004; 15: 4248-4260.

23. Torres M, Forman HJ. Redox signaling and the MAP kinase pathways. Biofactors 2003; 17: 287-296.

24. Hauet T, Mothes D, Goujon JM, Carretier M, Eugene M. Protective effect of polyethylene glycol against prolonged cold ischemia and reperfusion injury: study in the isolated perfused rat kidney. J Pharmacol Exp Ther 2001; 297: 946-952.

25. Mack JE, Kerr JA, Vreugdenhil PK, Belzer FO, Southard JH. Effect of polyethylene glycol on lipid peroxidation in cold-stored rat hepatocytes. Cryobiology 1991; 28: 1-7.

26. Abbas R, Kombu RS, Dignam D, Gunning W, Stulberg JJ, Brunengraber $\mathrm{H}$ et al. Polyethylene glycol modified-albumin enhances the cold preservation properties of University of Wisconsin solution in rat liver and a hepatocyte cell line. J Surg Res 2010; 164: 95-104.

27. Hotamisligil GS. Endoplasmic reticulum stress and the inflammatory basis of metabolic disease. Cell 2010; 140: 900-917.

28. Ben Mosbah I, Alfany-Fernández I, Martel C, Zaouali MA, Bintanel-Morcillo M, Rimola A et al. Endoplasmic reticulum stress inhibition protects steatotic and nonsteatotic livers in partial hepatectomy under ischemia-reperfusion. Cell Death Dis 2010; 1: e52.

29. Anderson CD, Upadhya G, Conzen KD, Jia J, Brunt EM, Tiriveedhi V et al. Endoplasmic reticulum stress is a mediator of posttransplant injury in severely steatotic liver allografts. Liver Transpl 2011; 17: 189-200.

30. Lim JH, Lee HJ, Ho Jung M, Song J. Coupling mitochondrial dysfunction to endoplasmic reticulum stress response: a molecular mechanism leading to hepatic insulin resistance. Cell Signal 2009; 21: 169-177.

31. Deniaud A, Sharaf el dein O, Maillier E, Poncet D, Kroemer G, Lemaire C et al. Endoplasmic reticulum stress induces calcium-dependent permeability transition, mitochondrial outer membrane permeabilization and apoptosis. Oncogene 2008; 27: 285-299.

32. Kitiphongspattana K, Khan TA, Ishii-Schrade K, Roe MW, Philipson LH, Gaskins HR. Protective role for nitric oxide during the endoplasmic reticulum stress response in pancreatic beta-cells. Am J Physiol Endocrinol Metab 2007; 292: E1543-E1554.

33. Eugene M. Polyethylene glycols and immunocamouflage of the cells tissues and organs for transplantation. Cell Mol Biol (Noisy-le-grand) 2004; 50: 209-215.

34. Hauet T, Goujon JM, Baumert H, Petit I, Carretier M, Eugene M et al. Polyethylene glycol reduces the inflammatory injury due to cold ischemia/reperfusion in autotransplanted pig kidneys. Kidney Int 2002; 62: 654-667.

35. Franco-Gou R, Mosbah IB, Serafin A, Abdennebi HB, Roselló-Catafau J, Peralta C. New preservation strategies for preventing liver grafts against cold ischemia reperfusion injury. J Gastroenterol Hepatol 2007; 22: 1120-1126.

36. Fernández L, Heredia N, Grande L, Gómez G, Rimola A, Marco A et al. Preconditioning protects liver and lung damage in rat liver transplantation: role of xanthine/xanthine oxidase. Hepatology 2002; 36: 562-572.

37. Kamada N, Calne RY. Orthotopic liver transplantation in the rat. Technique using cuff for portal vein anastomosis and biliary drainage. Transplantation 1979; 28: 47-50.

38. Steffen R, Krom RA, Ferguson D, Ludwig J. Comparison of University of Wisconsin (UW) and Eurocollins (EC) preservation solutions in a rat liver transplant model. Transpl Int 1990; 3: 133-136.

39. Puri P, Mirshahi F, Cheung O, Natarajan R, Maher JW, Kellum JM et al. Activation and dysregulation of the unfolded protein response in nonalcoholic fatty liver disease. Gastroenterology 2008; 134: 568-576.

40. Ben Mosbah I, Massip-Salcedo M, Fernández-Monteiro I, Xaus C, Bartrons R, Boillot $O$ et al. Addition of adenosine monophosphate-activated protein kinase activators to University of Wisconsin solution: a way of protecting rat steatotic livers. Liver Transp/2007; 13: $410-425$

41. Selzner N, Selzner M, Jochum W, Clavien PA. Ischemic preconditioning protects the steatotic mouse liver against reperfusion injury: an ATP dependent mechanism. J Hepatol 2003; 39: 55-61.

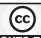

SOMERIAHIS

licensed under the Creative Commons Attribution-Noncommercial-No Derivative Works 3.0 Unported License. To view a copy of this license, visit http://creativecommons.org/licenses/by-nc-nd/3.0/ 